\section{Góndola, Enseñanza y Aprendizaje de las Ciencias}

DOI: https://doi.org/10.14483/23464712.16664
Resultado de Investigación

\title{
A FORMAÇÃO DE PROFESSORES EM ASTRONOMIA: UMA ANÁLISE DO BANCO DE TESES E DISSERTAÇÕES SOBRE EDUCAÇÃO EM ASTRONOMÍA*
}

\section{TEACHER EDUCATION IN ASTRONOMY: AN ANALYSIS OF THE BANK OF THESES AND DISSERTATIONS ON EDUCATION IN ASTRONOMY}

\section{LA FORMACIÓN DE PROFESORES EN ASTRONOMÍA: UN ANÁLISIS DEL BANCO DE ESTAS Y DISERTACIONES SOBRE LA EDUCACIÓN EN ASTRONOMÍA}

\begin{abstract}
Alexandre Shigunov Neto** (D) , Rodolfo Langhi
Cómo citar este artículo: Shigunov, A. y Langhi, R. (2021). A formação de professores em astronomia: uma análise do banco de teses e dissertações sobre educação em Astronomia. Góndola, enseñanza y aprendizaje de las ciencias, 16(3),

510-521. DOI: https://doi.org/10.14483/23464712.16664
\end{abstract}

\begin{abstract}
Resumo
Esta pesquisa pretende apresentar o mapeamento das teses e dissertações relacionados à temática da Formação de Professores em Educação em Astronomia disponibilizados no Banco de Teses e Dissertações sobre Educação em Astronomia. No levantamento de dados, realizado no site, foram identificadas 253 pesquisas que tratam especificamente da temática formação de professores em astronomia. As pesquisas foram divididas em seis categorias: Trabalho Docente, Políticas Públicas e Currículo, Formação Continuada de Professores, Formação Inicial de Professores, Concepções de Docência e Estado da Arte. Evidencia-se que a categoria mais investigada nas Teses e Dissertações defendidas até 2018 foi Trabalho Docente com 66\% do total de pesquisas.
\end{abstract}

Palavras Chave: Educação em Astronomia. Formação de professores. Ensino de Física. Estado da arte.

\begin{abstract}
This research intends to present the mapping of theses and dissertations related to the theme of Teacher Education in Astronomy made available at the Bank of Theses and Dissertations on Education in Astronomy. In the data survey carried out on the website, we identified 253 theses or dissertations that deal specifically with the theme of teacher education in astronomy. Six categories compound this research: Teaching Work, Public Policies and Curriculum, In-service Teacher
\end{abstract}

Recibido: 15 de julio de 2020; aprobado: 18 de mayo de 2021

* No Brasil a dissertação é o trabalho acadêmico que se destina à obtenção do grau de mestre em determinada área de conhecimento. Já a tese representa o trabalho final da pesquisa realizada no doutorado

** Mestre. Instituto Federal de Educação, Ciência e Tecnologia de São Paulo campus Itapetininga. Brasil. Email: shigunov@ifsp.edu.br. ORCID: https://orcid.org/0000-0002-0633-5237

*** Doutor. Programa de Pós-Graduação em Educação para a Ciência, Unesp Bauru. Brasil. Email: rodolfo.langhi@unesp.br. ORCID: https:// orcid.org/0000-0002-3291-5382 
Education, Pre-service Teacher Education, Teaching Conceptions, and the State of Art. It is evident that the most investigated category in these Theses and Dissertations developed until 2018 was Teaching Work with 66\% of the total research.

Keywords: Astronomy education. Teacher education. Physics teaching. State of the art.

\section{Resumen}

Esta investigación presenta un rastreo de tesis y disertaciones relacionadas con el tema de la Formación Docente en Astronomía, disponible en el Banco de Tesis sobre Educación en Astronomía. En la toma de datos realizada en el sitio web, se identificaron 253 tesis o disertaciones que tratan específicamente el tema de la formación del profesorado en Astronomía. La investigación se dividió en seis categorías: Trabajo docente, Políticas públicas y plan de estudios, Formación continua del profesorado, Formación inicial del profesorado, Concepciones docentes y Estado del arte. Es evidente que la categoría más investigada en las Tesis y Disertaciones defendidas hasta 2018 fue el Trabajo Docente con el 66\% de la investigación total.

Palabras clave: Educación en Astronomía. Formación del profesorado. Enseñanza de la física. Estado del arte.

\section{Introdução}

A educação em astronomia, enquanto objeto de estudo de pesquisadores brasileiros, começou a se desenvolver de forma sistemática e contínua a partir de 1999 com a publicação de dissertações, teses e artigos em periódicos. (IACHEL, 2009 e BRETONES; MEGID NETO, 2003). Porém, a primeira pesquisa de pós-graduação publicada referente à pesquisa em educação em astronomia se deu em 1973 com a tese de Caniato (1973).

De fato, desde 1999 a educação em astronomia tornou-se foco de diversos estudos de pesquisadores brasileiros, dentre os quais citamos Bretones e Compiani (2001), Bretones e Videira (2003), Bretones e Megid Neto (2003 e 2005), Langhi (2004), Megid Neto (2007), lachel e Nardi (2010), Langhi e Nardi (2009, 2012 e 2014) e Betrones (2011), Langhi (2018) e Shigunov Neto (2021).

Nesse direcionamento pretende-se verificar elementos que possam indicar: a) quais são os centros de pesquisa em educação em astronomia; b) quem são os orientadores de pós-graduação que atuam em educação em astronomia; c) o que se estuda em relação a temática da educação de astronomia (em outras palavras: Onde? Quem? O que?).

Há vários anos têm sido realizadas pesquisas do tipo estado da arte, tanto internacional quanto nacionalmente em diversas áreas de conhecimento, como ensino de ciências, formação de professores, ensino de Física, educação ambiental etc. Porém, em relação à Educação em Astronomia ainda há necessidade de estudos como este.

Portanto, este trabalho tem por objetivo principal mapear as teses e dissertações relacionadas à temática da Formação de Professores em Educação em Astronomia disponibilizados no Banco de Teses e Dissertações sobre Educação em Astronomia (BTDEA, 2020).

\section{As pesquisas do tipo estado da arte}

Esta pesquisa pode se caracterizar como estudo do tipo "estado da arte". Tais estudos possuem caráter bibliográfico e visam mapear e discutir a produção 
acadêmica em diferentes campos do conhecimento em determinado período de tempo (Ferreira, 2002; Salem, 2009 e 2012; Severino, 1986; Megid Neto, 1999; Cachapuz, 2003; Romanowski e Ens, 2006; Megid Neto, Carvalho, 2018; SHIGUNOV NETO, 2021).

Megid Neto e Carvalho (2018) analisaram os estudos denominados "estados da arte" ou "estado do conhecimento", os quais, segundo estes autores, podem:

ainda, ser entendidas como "metapesquisas" (pesquisa sobre pesquisas), uma vez que realizam novas compreensões ou interpretações de um determinado conjunto de trabalhos, e remetem à necessidade de investigações complementares. Não se restringem, apenas, à descrição do conjunto de pesquisas, vão além das pesquisas analisadas, buscando suas tendências, seus aspectos em comum, suas diferenças ou variabilidades. Dessa forma, conforme André, Simões, Carvalho e Brzezinski (1999) e André (2009), citados por Carvalho (2016), as pesquisas de estado da arte configuram-se, também, em trabalhos de "síntese integrativa" da produção científica em determinado campo de conhecimento, ou sobre determinada temática. Tal produção é desenvolvida em variadas instituições ou centros de pesquisa, em diversos períodos de tempo, sob diferentes referenciais teóricos e metodológicos, disponibilizadas por meio de inúmeros veículos ou recursos, enfim, uma produção de grande relevância que, muitas vezes, se encontra dispersa, pouco conhecida ou divulgada de modo insipiente ou inadequado. Os estudos de síntese integrativa podem, assim, reunir tal produção e promover sua descrição, interpretação, avaliação, trazendo novas luzes ao campo ou temática escolhidos, novas compreensões das pesquisas ali geradas, favorecendo uma divulgação mais adequada e um melhor conhecimento dos avanços (e eventuais limitações e lacunas) dessa produção. (Megid Neto e Carvalho, 2018, p.104-105)

Sendo assim, a presente pesquisa utilizará os estudos denominados de "Estado da Arte" ou "Estado do Conhecimento", por se tratar de um instrumento que busca a compreensão do conhecimento sobre determinado tema, em um período de tempo específico, e, consequentemente, sua sistematização e análise. Visando alcançar o objetivo proposto, elaboramos alguns questionamentos para nortear a metodologia do trabalho:

- Qual a quantidade de dissertações e teses defendidas entre 1973 e 2018 ?

- Quais as IES que possuem mais publicações sobre a temática Formação de Professores em Educação em Astronomia?

- Quem são os pesquisadores que mais orientam estudos sobre Formação de Professores em Educação em Astronomia no âmbito dos programas de pós-graduação?

- Quais os objetos de estudo ou temáticas pesquisadas no escopo dos trabalhos sobre Formação de Professores em Educação em Astronomia das teses e dissertações?

Para organizar os artigos de acordo com suas temáticas, adaptamos as categorias criadas por Brzezinski (2009) em um estudo do tipo estado da arte sobre formação de professores. As categorias criadas por Brzezinski (2009) foram utilizadas também por Gução, Jesus, Takahashi, Carnio e Nardi (2011), Carvalho e Shigunov Neto (2018) e Shigunov Neto (2021), as quais são:

- Concepções de Docência e de Formação de Professores - pesquisas que tratam de temáticas relacionadas com as abordagens teóricas oriundas da formação de professores e as características pedagógicas das concepções.

- Políticas Públicas e Currículo -trabalhos que englobam pesquisas sobre as políticas de formação docente, as políticas educacionais, as reformas educacionais e curriculares nos cursos de formação de professores e os currículos dos cursos de Ciências.

- Formação Inicial - investigações que tratam de questões referentes aos cursos de formação inicial de professores, com destaque para os estudos relativos aos cursos de licenciatura, pedagogia, estágios supervisionados.

- Formação Continuada - pesquisas dedicadas a estudar temáticas sobre o desenvolvimento profissional, pesquisa colaborativa, comunidades de 
prática, formação à distância, serviço de tutoria.

- Trabalho Docente - estudos que investigam aspectos associados à prática docente, saberes docentes, processo de ensino-aprendizagem, atuação docente em sala de aula.

- Revisão da Literatura - pesquisas que analisam trabalhos publicados em periódicos e/ou anais de eventos sobre a formação de professores, englobando também estudos do tipo "estado da arte".

\section{Instrumentos metodológicos}

O Banco de Teses e Dissertações sobre Educação em Astronomia (BTDEA) é um projeto de iniciativa do professor Paulo S. Bretones da UFSCar, disponibilizado a partir de 2010, apresentando teses e dissertações defendidas no Brasil sobre educação em astronomia no período compreendido entre 1973 e 2018. O BTDEA teve sua origem numa publicação por Bretones e Megid Neto (2005).

Importa destacar que, o recorte histórico de análise dessa pesquisa compreende o período de 1973 e 2018, pois o BTDEA apresenta dados atualizados até o ano de 2018.

Como nossa pesquisa versa sobre a temática da formação de professores em educação em astronomia, incluímos os termos "docente" e "professores" na busca realizada no BTDEA. As buscas foram realizadas por meio do sistema de busca do próprio site da BTDEA seguindo as seguintes etapas: 1) Pesquisa pelas palavras/expressões "docente" e "professores"; 2) Leitura dos resumos dos trabalhos; 3 ) Contagem e seleção dos trabalhos que mencionam Formação de Professores; 5) Análise qualitativa dos trabalhos selecionados por meio da leitura dos mesmos.

Do total de 448 documentos presentes no BTDEA, entre teses e dissertações que foram defendidas entre os anos de 1973 e 2018 nosso foco serão as 253 teses e dissertações que abordam a Formação de Professores em Educação em Astronomia.

\section{Resultados e análise}

Dentre os inúmeros Programas de Pós-Graduação do país, selecionamos para a análise as pesquisas produzidas em programas de pós-graduação acadêmicos e programas de pós-graduação profissionais autorizados pela CAPES.

Na área de Astronomia na CAPES atualmente há seis programas:

- Instituto Nacional de Pesquisas Espaciais (INPE) M e D

- Observatório Nacional (ON) - M e D

- Universidade Cidade de São Paulo (UNICID) - M e D

- Universidade de São Paulo (US) - M e D

- Universidade Federal do Espírito Santo (UFES) - D

- Universidade Federal do Rio de Janeiro (UFRJ) - M e D

A área de Física na CAPES conta atualmente conta com três programas:

- Mestrado Profissional em Ensino de Física - PROFIS, coordenado pela Sociedade Brasileira de Física.

- Programa de Pós-Graduação em Física e Astronomia da Universidade do Vale do Paraíba - UNIVAP.

Tabela 1. As produções na área de educação em astronomia entre as décadas de 1970 e 1990

\begin{tabular}{|c|c|c|c|c|}
\hline Ano & TES & Nível & Autor & Título \\
\hline 1973 & UNESP & $\mathrm{D}$ & Rodolpho Caniato & Um Projeto Brasileiro para o Ensino de Física \\
\hline 1986 & UNICAMP & $\mathrm{M}$ & Marcos César Danhoni & Astronomia de régua e compasso: de Kepler a Ptolomeu \\
\hline 1990 & USP & $\mathrm{M}$ & $\frac{\text { Silvania Sousa do }}{\text { Nascimento }}$ & Um curso de gravitação para professores de primeiro grau \\
\hline 1996 & UNICAMP & $\mathrm{D}$ & Maurício Compiani & $\begin{array}{l}\text { As Geociências no ensino fundamental: um estudo de caso sobre o } \\
\text { tema "A formacão do Universo" }\end{array}$ \\
\hline 1997 & UNESP & $\mathrm{M}$ & Ana Isabel Cardoso da Silva & $\begin{array}{l}\text { Um estudo sobre a aplicabilidade do currículo básico de ciências } \\
\text { para a escola pública do Paraná }\end{array}$ \\
\hline 1998 & USP & $\mathrm{D}$ & Sérgio Mascarello Bisch & $\begin{array}{l}\text { Astronomia no ensino fundamental: natureza e conteúdo do } \\
\text { conhecimento de estudantes e professores }\end{array}$ \\
\hline 1998 & UNESP & $\mathrm{M}$ & Nelson José Peruzzi & Modelos geocéntricos de Platáo à Ptolomeu: uma contribuição para \\
\hline 1998 & UFMT & $\mathrm{M}$ & Tânia Maria Lima Beraldo & $\begin{array}{l}\text { Oéries iniciais do ensino fundamental: elementos para reflexão em } \\
\text { torno da formacão docente }\end{array}$ \\
\hline
\end{tabular}

Fonte: BTDEA (2020). 


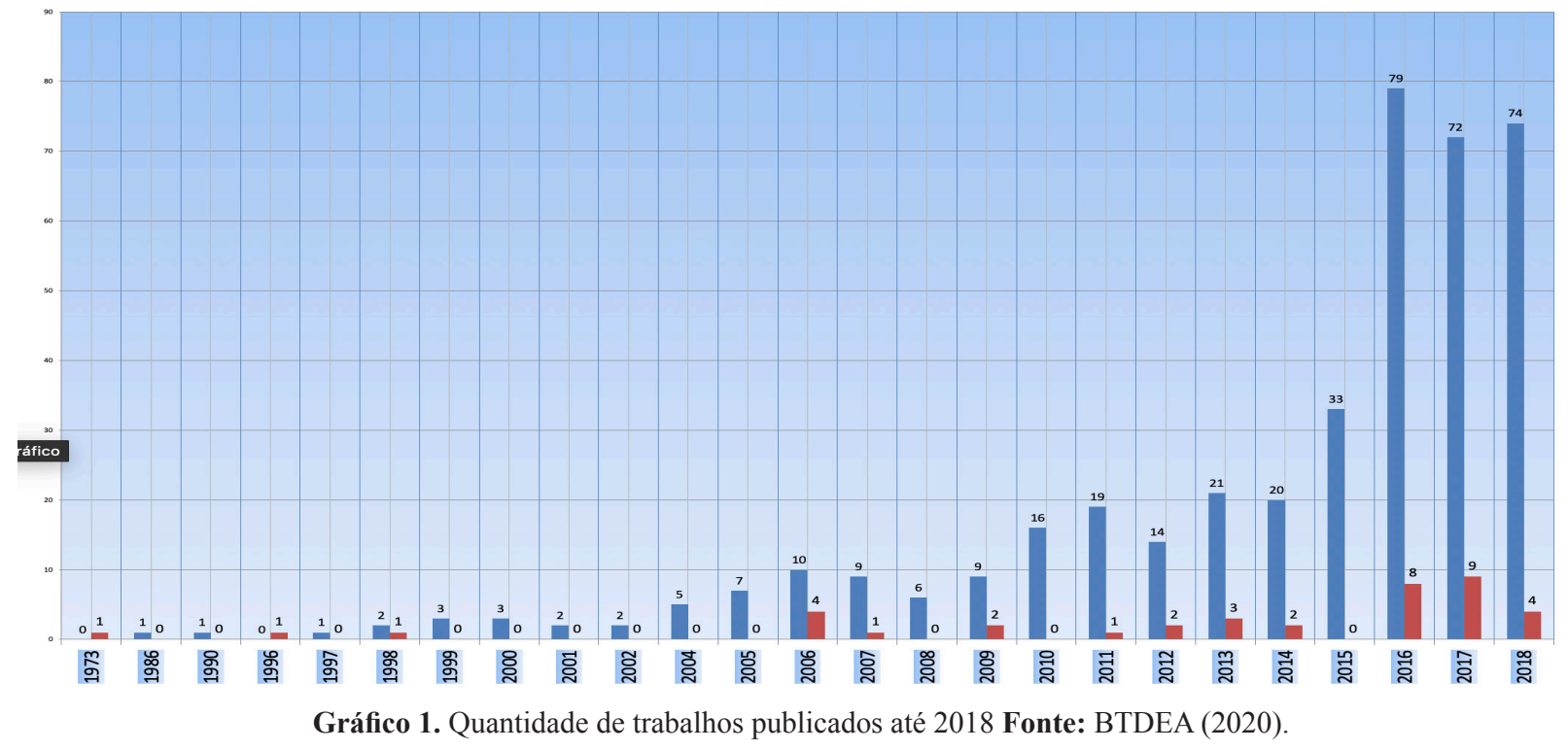

- Programa de Pós-Graduação em Física e Astronomia da Universidade Federal Tecnológica Federal do Paraná - UTFPR.

Na área de Ensino são 230 cursos de pós-graduação, muitos de Ensino de Ciências e Matemática, e um curso específico de Mestrado Profissional em Ensino na área de Astronomia da Universidade Estadual de Feira de Santana (UEFS).

O BTDEA tem cadastrado atualmente 448 pesquisas, sendo 409 dissertações e 39 teses defendidas em programas de pós-graduação no período de 1973 e 2018.

A primeira pesquisa na área de Educação em Astronomia data de 1973, a tese de doutorado do pesquisador Rodolpho Caniato defendida na Universidade Estadual Paulista de Rio Claro com o título "Um Projeto Brasileiro para o Ensino de Física", orientada por José Goldemberg. Essa é a primeira pesquisa na área de Educação em Astronomia no Brasil (BRETONES, MEGID NETO, 2005; BRETONES, 2011; LANGHI, 2018).

Somente treze anos após a primeira pesquisa ter sido publicada na área é que o pesquisador Marcos César Danhoni Neves defende sua dissertação de mestrado na UNICAMP sob a orientação do professor Carlos A. Arguello.

A tabela 1 apresenta as defesas nas três primeiras décadas da pesquisa em educação em astronomia no
Brasil - 1970, 1980 e 1990. As oito primeiras pesquisas da área de Educação em Astronomia no Brasil, ocorreram num período de 25 anos e foram cinco dissertações de mestrado e três teses de doutorado. As três teses de doutorado foram defendidas na Universidade Estadual Paulista, Universidade Estadual de Campinas e Universidade de São Paulo. Já as cinco dissertações também foram defendidas nas três Universidades Estaduais do Estado de São Paulo, com uma exceção, que ocorreu na Universidade Federal de Mato Grosso.

Fato de destaque é que dos oito pesquisadores que defenderam suas teses e dissertações nos primeiros vinte e cinco anos, apenas o professor Sérgio Marcarello Bisch atua com Educação em Astronomia, conforme consta do CV Lattes.

O gráfico 1 exibe a produção da área de Educação em Astronomia no Brasil no período compreendido entre 1973 e 2018, de dissertações e teses.

Pelo gráfico 1 é possível perceber que até o ano de 2009, com ressalva do ano de 2006 que teve dez dissertações, as defesas de mestrado e doutorado não ultrapassavam um dígito. De 2010 a 2015 os mestrados defendidos oscilam entre 16 e 20, e a partir de 2015 há um salto significativo de defesas. Os três anos com mais teses defendidas foram: 2006 e 2018 com quatro, 2016 com oito trabalhos e 2017 com nove teses. No que se refere às dissertações 
os anos com mais produções foram: 2016 com 79 defesas, 2017 com 72 dissertações defendidas e 2018 com 74 defesas.

O gráfico 1 revela que o crescimento nas defesas de mestrado entre 2015 e 2014 foi de $65 \%$, no ano seguinte 2016 a expansão foi extraordinária, ultrapassando 139,39\%. Em 2017 houve um decréscimo de $9,72 \%$ e em 2018 um aumento de $2,77 \%$.

Por fim, cabe registrar que o ano de 2016 foi o ano com maior número de defesas ocorridas nos programas de pós-graduação no Brasil, com 79 mestrados e oito doutorados.

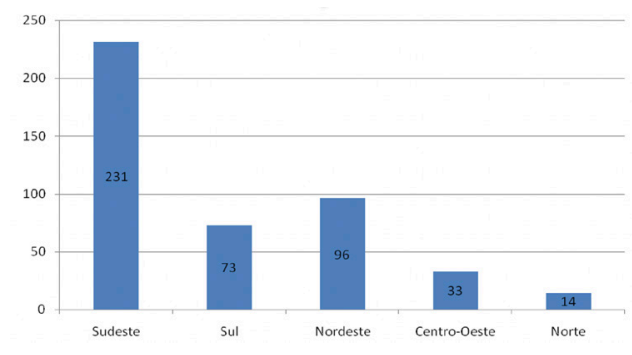

Gráfico 2. Quantidade de pesquisas por região. Fonte: BTDEA (2020).

Pelo gráfico 2 constata-se que a região Sudeste responde por $51,56 \%$ do total da produção nacional no período sobre Educação em Astronomia. Contudo,

Tabela 2. Pesquisadores com mestrado e doutorado em Educação em Astronomia.

\begin{tabular}{|c|c|c|c|c|}
\hline & Orientador & IES & Nível & Ano \\
\hline \multirow{2}{*}{ Carlos Aparecido Kantor } & Luis Carlos de Menezes & USP & $\mathrm{D}$ & 2012 \\
\hline & Luis Carlos de Menezes & USP & M & 2001 \\
\hline \multirow[t]{2}{*}{ Cristina Leite } & YassukoHosoume & USP & $\mathrm{D}$ & 2006 \\
\hline & YassukoHosoume & USP & M & 2002 \\
\hline \multirow[t]{2}{*}{ Denis Eduardo Peixoto } & Maurício UrbanKleinke & UNICAMP & $\mathrm{D}$ & 2018 \\
\hline & Maurício UrbanKleinke & UNICAMP & M & 2013 \\
\hline \multirow[t]{2}{*}{ Edson Pereira Gonzaga } & Marcos RinconVoelzke & UNICSUL & D & 2016 \\
\hline & Marcos RinconVoelzke & UNICSUL & M & 2009 \\
\hline \multirow{2}{*}{ Evonir Albrecht } & Marcos RinconVoelzke & UNICSUL & D & 2012 \\
\hline & Marcos RinconVoelzke & UNICSUL & M & 2008 \\
\hline \multirow{2}{*}{ Gustavo lachel } & Roberto Nardi & UNESP & D & 2013 \\
\hline & Roberto Nardi e Rosa Maria Fernandes Scalvi & UNESP & M & 2009 \\
\hline \multirow{2}{*}{ Joana Brás Varanda Marques } & Denise de Freitas & UESCar & $\mathrm{D}$ & 2017 \\
\hline & Denise de Freitas & UESCar & M & 2014 \\
\hline \multirow{2}{*}{ Leonardo Marques Soares } & Silvania Sousa do Nascimento & & $\mathrm{D}$ & 2017 \\
\hline & Silvania Sousa do Nascimento & UFMG & M & 2010 \\
\hline \multirow{2}{*}{ Michel Paschini Neto } & Maria Guiomar Carneiro Tommasiello & UNIMEP & $D$ & 2016 \\
\hline & Maria Guiomar Carneiro Tommasiello & UNIMEP & M & 2011 \\
\hline \multirow{2}{*}{ Paulo Henrique Azevedo Sobreira } & Maria Elena Ramos Simielli & USP & $\mathrm{D}$ & 2006 \\
\hline & Maria Elena Ramos Simielli_ & USP & M & 2002 \\
\hline \multirow{2}{*}{ Paulo Sergio Bretones } & Maurício Compiani & UNICAMP & $\mathrm{D}$ & 2006 \\
\hline & Maurício Compiani & UNICAMP & M & 1999 \\
\hline \multirow[t]{2}{*}{ Roberta ChiesaBartelmebs } & João Batista Siqueira Harres & PUCRS & $\mathrm{D}$ & 2016 \\
\hline & Roque Moraes & FURG & M & 2012 \\
\hline \multirow[t]{2}{*}{ Rodolfo Langhi } & Roberto Nardi & UNESP & $\mathrm{D}$ & 2009 \\
\hline & Roberto Nardi & UNESP & M & 2004 \\
\hline \multirow{2}{*}{ VitéricoJabur Maluf } & Vera Teresa Valdemarin & UNESP & $\mathrm{D}$ & 2006 \\
\hline & José Adolfo Rodriguez & UEMT & M & 2000 \\
\hline
\end{tabular}

Fonte: BTDEA (2020). 
fato de destaque é a região nordeste ter uma quantidade maior de defesas do que a região Sul. Uma suposição é que essa surpreendente segunda colocação da região nordeste ocorra por existirem alguns centros de pesquisa que atuam em Educação em Astronomia, tais como o Mestrado Profissional em Astronomia da Universidade Estadual de Feira de Santa e a Universidade Federal do Rio Grande do Norte.

A tabela 2 mostra os pesquisadores que defenderam o mestrado e o doutorado na área de Educação em Astronomia no período compreendido entre 1973 e 2018.

Ao todo foram 14 pesquisadores que finalizaram o mestrado e doutorado com pesquisas sobre Educação em Astronomia no Brasil. Desses, apenas 2 pesquisadores concluíram suas pesquisas de mestrado e doutorado em IES diferentes e com orientadores diferentes.

Dos pesquisadores que concluíram os cursos de mestrado e doutorado com pesquisas na área de Educação em Astronomia todos continuam atuando na área, mas 3 não atuam em programas de pós-graduação de IES.

O gráfico 3 exibe um comparativo entre as pesquisas de Educação em Astronomia e as pesquisas sobre a Formação de Professores em Educação em Astronomia no período analisado.
Interessante notar que até o ano de 2010 a temática da formação de professores dominava as pesquisas, em alguns anos tendo todos os trabalhos versando sobre essa temática. Além disso, este assunto responde $56,70 \%$ do total de publicações defendidas no período analisado.

O gráfico 4 retrata os pesquisadores com mais orientações no período sobre Formação de Professores e Educação em Astronomia.

Os três pesquisadores com mais orientações defendidas sobre Formação de Professores e Educação em Astronomia pertencem ao quadro docente da Universidade Cruzeiro do Sul, Universidade Estadual Paulista de Bauru, Universidade Estadual de Feira de Santana. Algumas dissertações e teses possuem dois pesquisadores, sugerindo o papel do coorientador. O gráfico 5 retrata um panorama das IES com Programas de Pós-Graduação que mais publicações obtiveram no período pesquisado sobre a temática analisada, tanto no nível de mestrado, quanto em doutorado.

Das sete IES com mais orientações temos quatro da região Sudeste, duas da região Nordeste e uma da região Sul, fato que valida os dados representados pelo gráfico 2 em que a região Sudeste é o principal polo de formação de pesquisadores.

A Universidade de São Paulo (USP) surge como o principal centro de formação de pesquisadores na

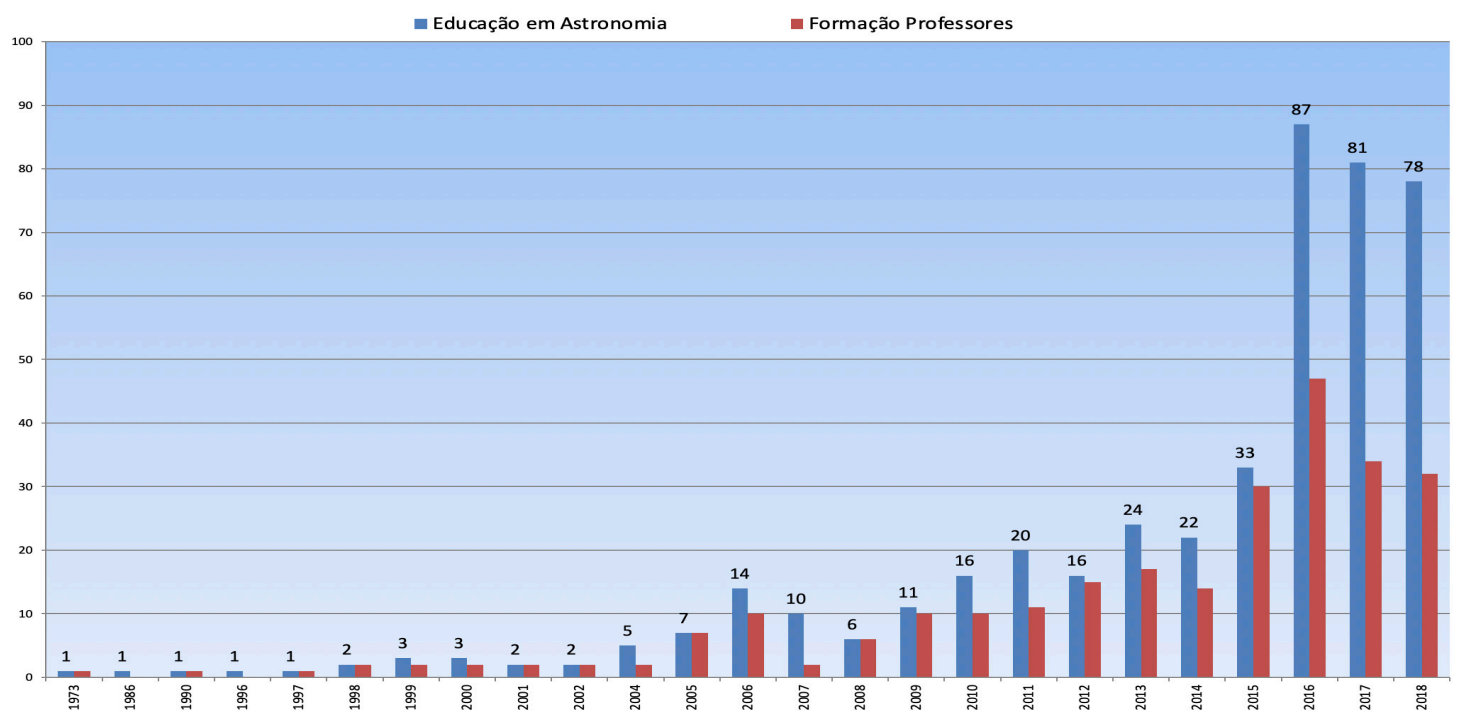

Gráfico 3. Comparativo entre pesquisas em Educação em Astronomia e Formação de Professores em Educação em Astronomia.

Fonte: BTDEA (2020). 


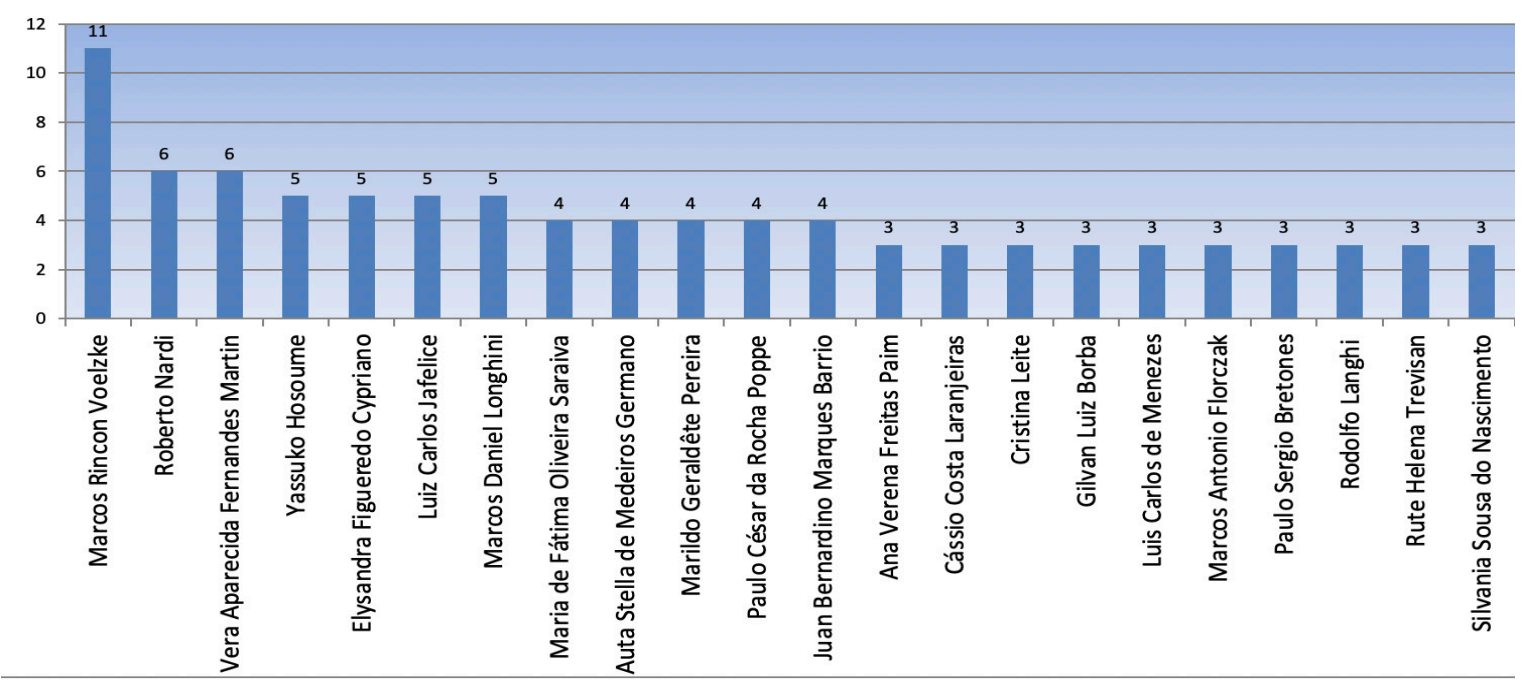

Gráfico 4. Pesquisadores com mais orientações.Fonte: BTDEA (2020).

área Formação de Professores e Educação em Astronomia, com 34 trabalhos defendidos no decorrer de 45 anos. Importa destacar que na USP temos ao menos três Programas de Pós-Graduação que podem produzir material sobre a temática: Educação, Interunidades em Ensino de Ciências e Astronomia e Astrofísica. Entre os orientadores com mais trabaIhos estão quatro pesquisadores desta universidade. A UNESP de Bauru vem se destacando no cenário nacional com seu programa de 15 anos de existência. Apenas 13 anos após a primeira defesa, já se encontra entre os principais formadores de pesquisadores da área de Ensino de Ciências e também em Formação de Professores e Educação em Astronomia. Em grande parte, tal destaque pode ser atribuído a dois pesquisadores ali atuantes, que respondem por $47,37 \%$ do total de orientações.
A UEFS com seu Mestrado Profissional em Astronomia é a terceira com mais orientações e a mais nova das IES, cujo programa fora criado em 2013. Nesta universidade, são três pesquisadores que respondem por $87,50 \%$ do total de trabalhos defendidos.

Há mais dois Programas de IES que podem ser destacados. Um deles é o Programa de Pós-Graduação em Ensino de Ciências Naturais e Matemática da Universidade Federal do Rio Grande do Norte (UFRN) com 15 trabalhos orientados por três pesquisadores. O Mestrado Profissional em Ensino de Ciências e Matemática da Universidade Cruzeiro do Sul (UNICSUL) foi a quinta IES com mais defesa de dissertações e teses sobre Formação de Professores e Educação em Astronomia, sendo apenas um pesquisador o responsável por quase $80 \%$ do total das orientações.

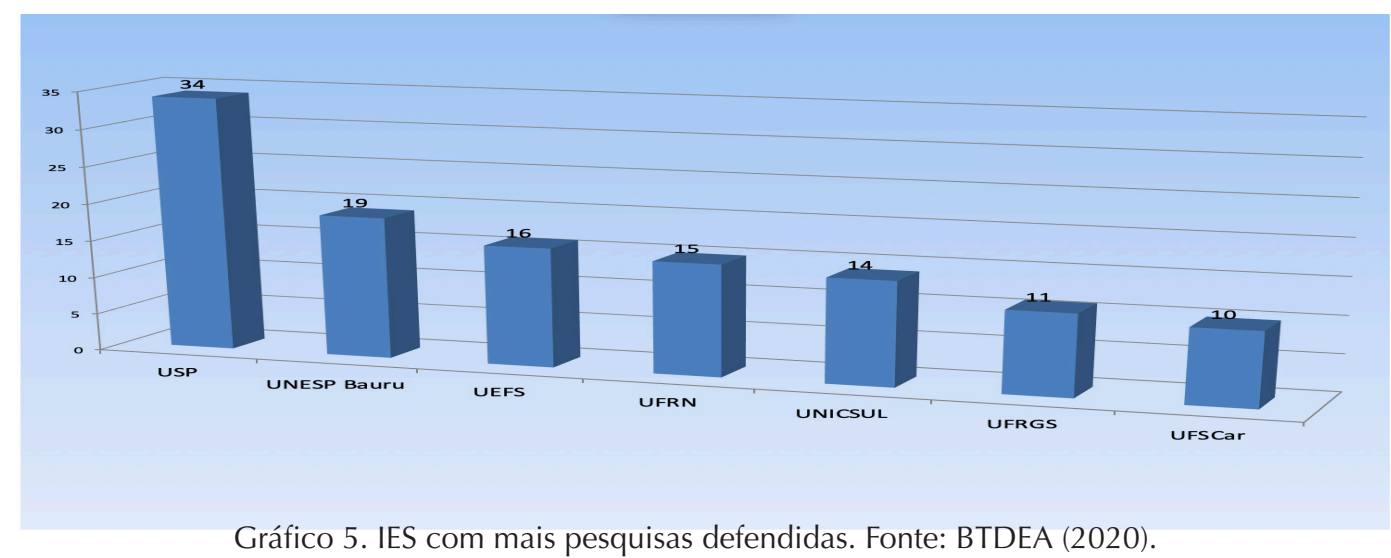




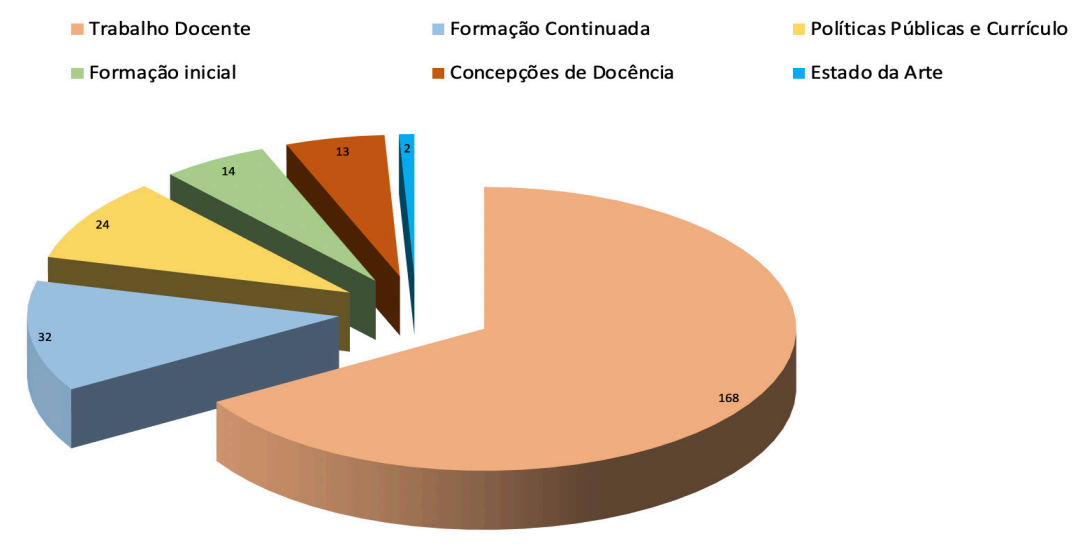

Gráfico 6. Temáticas mais presentes nas teses e dissertações defendidas no âmbito da Formação de Professores. Fonte: BTDEA (2020).

Outras IES também podem ser citadas por possuírem alguns trabalhos na área de Educação em Astronomia: Universidade Estadual de Londrina, Universidade Metodista de Piracicaba, Universidade Federal de Santa Catarina, Universidade Federal de Goiás, Universidade Estadual de Campinas, Universidade de Brasília, Universidade Federal de Uberlândia, Universidade Estadual de Maringá, Universidade Federal do ABC e Universidade Federal de Minas Gerais. Também se encontram trabalhos defendidos em mestrados profissionais em Institutos Federais de Educação, Ciência e Tecnologia.

O gráfico 6 demonstra a distribuição dos 253 trabaIhos sobre Formação de Professores em Educação em Astronomia, em que 66\% discutem o Trabalho Docente, relacionados a aspectos associados à prática docente, saberes docentes, processo de ensino-aprendizagem, atuação docente em sala de aula. A temática da Formação Continuada de Professores foi a segunda categoria com mais pesquisas, cujas temáticas tratam sobre o desenvolvimento profissional, pesquisa colaborativa, comunidades de prática, formação à distância, serviço de tutoria.

A temática Políticas Públicas e Currículo engloba pesquisas sobre as políticas de formação docente, as políticas educacionais, as reformas educacionais e curriculares nos cursos de formação de professores e os currículos dos cursos de Ciências.

Presente em 14 pesquisas defendidas, a categoria
Formação Inicial de Professores trata de questões referentes aos cursos de formação inicial de professores, com destaque para os estudos relativos aos cursos de licenciatura, pedagogia e estágios supervisionados.

A categoria Concepções de Docência, presente em 13 das pesquisas, trata de temáticas relacionadas com as abordagens teóricas oriundas da formação de professores e as características pedagógicas das concepções.

E por fim, com apenas duas citações, Estado da Arte ou Revisão da literatura, envolve estudos bibliográficos cujas fontes de pesquisa são periódicos e/ou anais de eventos sobre a formação de professores, englobando também estudos do tipo "estado da arte" ou "estado do conhecimento".

Portanto, pela análise quantitativa realizada nas 253 teses e dissertações do Banco de Teses e Dissertações sobre Educação em Astronomia (BTDEA) que abordam a Formação de Professores em Educação em Astronomia foi possível constatar uma forte tendência de publicações sobre as seguintes temáticas, nesta ordem: Trabalho Docente, Políticas Públicas e Currículo e Formação de Continuada de Professores.

\section{Conclusões}

Acredita-se que ao finalizar a investigação sobre as pesquisas da área de Formação de Professores em Educação de Astronomia no Banco de Teses e 
Dissertações sobre Educação em Astronomia (BTDEA) foi possível alcançar os objetivos previamente pretendidos e verificar indicadores que pudessem responder aos questionamentos iniciais: Quais são os centros de pesquisa em Formação de Professores em Educação em Astronomia? Quem são os pesquisadores que atuam mais fortemente na área? O que se estuda em relação a temática da Formação de Professores em Educação em Astronomia.

As seis categorias identificadas neste levantamento foram: Trabalho Docente, Políticas Públicas e Currículo, Formação Continuada de Professores, Formação Inicial de Professores, Concepções de Docência e Estado da Arte. Evidencia-se que a categoria mais investigada nas teses e dissertações defendidas até 2018 foi Trabalho Docente com 66\% do total de pesquisas.

Pelos dados extraídos do BTDEA, as IES que mais produzem sobre Formação de Professores e Educação em Astronomia são: USP (Programas de Educação, Interunidades em Ensino de Ciências e Astronomia e Astrofísica), Programa de Pós-Graduação em Educação para a Ciência da UNESP de Bauru,Programa de Mestrado Profissional em Astronomia da UEFS,Programa de Pós-Graduação em Ensino de Ciências Naturais e Matemática da Universidade Federal do Rio Grande do Norte (UFRN), Universidade Federal do Rio Grande do Norte (UFRN) e o Mestrado Profissional em Ensino de Ciências e Matemática da Universidade Cruzeiro do Sul (UNICSUL).

Os dados coletados demonstram que os anos de 2015, 2016, 2017 e 2018 foram os que apresentaram maior quantidade de defesas, tanto na temática da Educação em Astronomia quanto em Formação de Professores em Educação em Astronomia. Outro resultado de destaque é que até o ano de 2009 a categoria Formação de Professores correspondia a praticamente todas as pesquisas da área, sendo que a partir de 2010 passou a ocupar cerca da metade das produções.

Pela análise quantitativa constata-se que há uma tendência de publicações sobre Formação de Professores em Educação em Astronomia sobre as seguintes temáticas nesta ordem de interesse: Trabalho Docente, Formação Continuada de Professores e Políticas Públicas e Currículo.

Outras sugestões de pesquisas que podem ser desenvolvidas sobre categorias pouco exploradas, são: políticas e propostas de profissionais da educação em astronomia, identidade e profissionalização docente, revisão da literatura, métodos e técnicas de ensino. Trabalhos como estes potencializam outras questões importantes para o aprimoramento do debate sobre a Formação de Professores em Educação em Astronomia.

Esperamos que estas e outras reflexões possam ser ampliadas e que os estudos sobre a Formação de Professores em Educação em Astronomias e proliferem, em quantidade e qualidade.

\section{Referências}

BRETONES, P. S. \& MEGID NETO, J., Tendências de teses e dissertações sobre ensino de Astronomia no Brasil, In: REUNIÃO ANUAL DA SOCIEDADE ASTRONÔMICA BRASILEIRA, XXIX, 2003, Águas de São Pedro.

BRETONES, P. S. Banco de Teses e Dissertações sobre Educação em Astronomia: implantação, dificuldades e possíveis contribuições. 2011. In: SIMPÓSIO NACIONAL DE EDUCAÇÃO EM ASTRONOMIA, 1., 2011, Rio de Janeiro. Disponível em: https://sab-astro.org.br/wp-content/uploads/2017/03/SNEA2011 TCO15. pdf. Acesso em 20 maio. 2020.

BRETONES, Paulo Sergio; COMPIANI, Maurício. Disciplinas Introdutórias de Astronomia nos Cursos Superiores do Brasil. Boletim da Sociedade Astronômica Brasileira. v. 20, n. 3, p. 61-82, 2001.

BRETONES, Paulo Sergio; MEGID NETO, Jorge. Tendências de Teses e Dissertações sobre Educação em Astronomia no Brasil. Boletim da Sociedade Astronômica Brasileira. v. 24, n. 2, p. 35-43, 2005.

BRETONES, Paulo Sergio; VIDEIRA, Antonio Augusto Passos. A Astronomia no Ensino Superior 
Brasileiro entre 1808 e 1889. Boletim da Sociedade Astronômica Brasileira. v. 22, n. 3, p. 37-49, 2003.

BRZEZINSKI, I. Formação de profissionais da educação no GT 8/Anped. : travessia histórica. Revista Brasileira de Pesquisa sobre Formação de Professores, v. 1, p. 1-5, 2009.

CACHAPUZ, António F. Do sentido actual da pesquisa em formação de professores de ciências. In: ENCONTRO DE PESQUISA EM EDUCAÇÃO EM CIÊNCIAS, IV, Bauru, 2003. Atas... 2003, CDROM.

CANIATO, Rodolpho. Um projeto brasileiro para o ensino de Física. Rio Claro. Universidade Estadual Paulista. UNESP, 1973. 586p. Tese de Doutorado.

CARVALHO, Raquel Aparecida e SHIGUNOV NETO, Alexandre. Uma visão da pesquisa sobre formação de professores no Brasil presente em periódicos da área de educação: análise da produção acadêmica entre os anos de 2000 e 2017. Revista Brasileira de Iniciação Científica. Itapetininga. Vol. 5, n. 3, 2018.

FERREIRA, Norma Sandra de Almeida. As pesquisas denominadas "estado da arte". Educ. Soc., Campinas, v. 23, n. 79, p. 257-272, ago. 2002.

GUÇÃO, Maria Fernanda Bianco; JESUS, Andréa Cristina Souza de; TAKAHASSHI, Bruno Tadashi; CARNIO, Michel Pisa e NARDI, Roberto. Um panorama sobre a temática da formação de professores de física presente em periódicos da área de ensino de ciências na última década. XIX SIMPÓSIO NACIONAL DE ENSINO DE FÍSICA (SNEF), Manaus, 2011,

IACHEL, G. Um estudo exploratório sobre o ensino de Astronomia na formação continuada de professores. 2009. 229 f. Dissertação (Mestrado em Educação para a Ciência). Faculdade de Ciências, UNESP, Bauru, 2009.

IACHEL, Gustavo e NARDI, Roberto. Algumas tendências das publicações relacionadas à astronomia em periódicos brasileiros de ensino de Física nas últimas décadas. Ens. Pesqui. Educ. Ciênc. (Belo Horizonte), Ago 2010, vol.12, no.2, p.225-238. ISSN 1983-2117.

LANGHI, R. Um estudo exploratório para a inserção da Astronomia na formação de professores dos anos iniciais do ensino fundamental. 2004. 240 f. Dissertação (Mestrado em Educação para a Ciência). Faculdade de Ciências, UNESP, Bauru, 2004

Langhi, R., \& Nardi, R. Justificativas para o ensino de Astronomia: o que dizem os pesquisadores brasileiros?. Revista Brasileira de Pesquisa em Educação em Ciências, . 14, n. 3, 041-059, 2014.

LANGHI, Rodolfo e NARDI, Roberto. Ensino de astronomia no Brasil: educação formal, informação, não formal e divulgação científica. Revista Brasileira de Ensino de Física, v. 31, n. 4, 4402, 2009.

LANGHI, Rodolfo. Teses e Dissertações. Disponível em: https://sites.google.com/site/ proflanghi/resumos. Acesso em 01 jun. 2020.

LANGHI, Rodolfo; NARDI, Roberto. Educação em Astronomia: repensando Formação de professores. São Paulo: Escrituras, 2012

MEGID NETO, J. Tendências da pesquisa acadêmica sobre o ensino de Ciências no nível fundamental. 1999. 365 p. Tese (Doutorado em Educação) - Faculdade de Educação, Universidade Estadual de Campinas, Campinas, 1999.

MEGID NETO, J. Três décadas da pesquisa em educação em Ciências: tendências em teses e dissertações (1972-2003). In: NARDI, R. (org). A pesquisa em ensino de Ciências no Brasil: alguns recortes. p. 341-356. São Paulo: Escrituras Editora, 2007

MEGID NETO, Jorge. Tendências da pesquisa acadêmica sobre o ensino de Ciências no nível fundamental. Campinas: Faculdade de Educação, Universidade Estadual de Campinas, 1999. 365p. (Tese de doutorado).

MEGID NETO, Jorge; CARVALHO, Luiz Marcelo. Pesquisas de estado da arte: fundamentos, características e percursos metodológicos. In: ESCHENHAGEN, G. M. L.; VÉLEZ-CUARTAS, G. MALDONADO, C.; PINO, G.G (Edits). 
Construcción de problemas de investigación: diálogos entre el interior y el exterior. Universidad Pontifica Bolivariana / Universidad de Antioquia: Medellin, 2018. p. 97-113.

ROMANOWSKI, JoanaPaulin e ENS, Romilda Teodora. AS PESQUISAS DENOMINADAS DOTIPO "ESTADO DA ARTE" EM EDUCAÇÃO. Revista Diálogo Educacional, [S.I.], v. 6, n. 19, p. p. 37-50, jul. 2006. ISSN 1981-416X. Disponível em: $<$ https://periodicos.pucpr.br/index.php/dia-

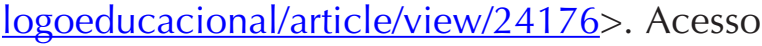
em: 20 maio. 2020.

SALEM, Sonia. Estado da arte dos estados da arte da pesquisa em ensino de Física. VII ENCONTRO NACIONAL DE PESQUISAS EM EDUCAÇÃO DE CIÊNCIAS. Florianópolis, 2009.

SALEM, Sonia. Perfil, evolução e perspectivas da pesquisa em ensino de física no Brasil. Tese de doutorado. Universidade de São Paulo, São Paulo, 2012.

SEVERINO, Antonio J. Metodologia do trabalho científico. São Paulo: Cortez \& Moraes, 1986.

SHIGUNOV NETO, A. O que se pesquisa em educação em astronomia: uma análise do periódico Revista Latino-Americana de Educação em Astronomia no período compreendido de 2004 a 2019. Revista Internacional de Pesquisa em Didática das Ciências e Matemática, [S. I.], v. 2, p. e021002, 2021. Disponível em: https://periodicoscientificos.itp.ifsp.edu.br/index.php/revin/ article/view/336. Acesso em: 05 maio. 2021. 